\title{
Next Generation IR Magnets for Hadron Colliders
}

\author{
R. Gupta, M. Anerella, J. Cozzolino, J. Escallier, G. Ganetis, M. Harrison and P. Wanderer
}

\begin{abstract}
Brookhaven National Laboratory (BNL) is developing "React \& Wind" designs and technology for building long high field accelerator magnets. This paper presents the R\&D program for interaction region (IR) magnets made with "Rutherford" cable for the luminosity upgrade of the Large Hadron Collider (LHC). This paper will introduce a few new end design concepts that make the bend radius of the cable in the end independent of the coil aperture. These designs are suitable for building magnets with "React \& Wind" technology.
\end{abstract}

Index Terms. Accelerators, Interaction Region, React \& Wind, Quadrupoles, Superconducting Magnets.

\section{INTRODUCTION}

A multi-lab accelerator $R \& D$ program has been proposed for developing next generation interaction region (IR) for the luminosity upgrade of Large Hadron Collider, now under construction [1]. A common feature of all upgrade scenarios is the need for magnets with a large pole tip field. The superconductors that can generate usable field above $10 \mathrm{~T}$, such as $\mathrm{Nb}_{3} \mathrm{Sn}$ and HTS (see Fig. 1), are brittle in nature. The Superconducting Magnet Division at Brookhaven National Laboratory has opted to develop "React \& Wind" magnet technology for high field accelerator magnets [2]. The "React $\&$ Wind" approach eliminates the requirement of dealing with the differential thermal expansions of the various materials of coil modules during reaction in the "Wind and React" approach - a requirement that becomes more demanding as magnets get longer. The "React \& Wind" approach also allows one to use a variety of insulation and other materials since the coil and its associated structure are not subjected to the high reaction temperature. Moreover, if successful, one can benefit from using the techniques and tooling developed for building $\mathrm{NbTi}$ magnets. For these reasons a successful demonstration of "React \& Wind" technology will be a major asset for future $\mathrm{Nb}_{3} \mathrm{Sn}$ magnets. The "React \& Wind" approach is even more relevant to HTS magnets where the required temperature control must be $\sim 0.5$ degree at $\sim 880$ degree Celsius over the entire coil volume.

Manuscript received August 4, 2002.

The authors are with Brookhaven National Laboratory, Building 902A, Superconducting Magnet Division, Upton, NY 11973 USA.

Corresponding author: Ramesh Gupta (phone: 631-344-4805; fax: 631-3442190; e-mail: gupta@bnl.gov).

Work supported by the U.S. Department of Energy under contract No. DEAC02-98CH10886.

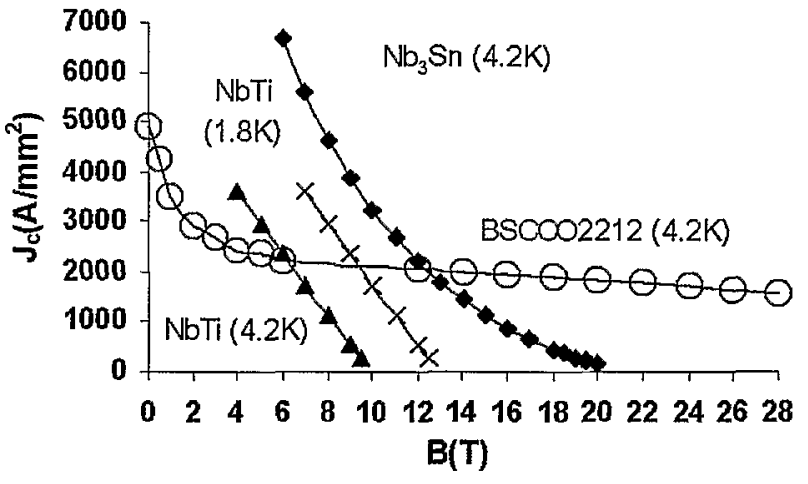

Fig 1: The performance of conductor in year 2000.

The "React \& Wind" approach, however, requires one to deal with the brittle pre-reacted cable during coil winding without causing large degradation. The LHC upgrade is likely to take place around year 2015. BNL considers that at this stage it would be prudent to investigate various options before committing to a particular technology or to a set of design parameters. This is particularly important since there has been limited experience with the relatively new technology that is the basis for the design. This would allow beam physicists more time to study and develop a more optimized IR lattice (and magnet design parameters) that would benefit from the initial operating experience of the current LHC interaction region.

The first phase of the BNL program would focus on developing designs and technologies. The designs presented here are generic in nature. The goal of the program is to produce quadrupole magnets with an operating pole tip field of 11-14 Tesla and a coil aperture of 50-90 mm.

\section{END DESIGNS FOR REACT \& WIND MAGNETS}

Brittle high field superconductors are prone to degradation when the bending strain exceeds a critical value. Therefore, the ends, where the cable must bend, should drive the conceptual design of the "React and Wind" high field magnets. A simple 2-in-1 common coil dipole concept is an example of this philosophy [3]. An elegant equivalent of a simple coil geometry is not yet known for a single aperture quadrupole magnet. However, the end designs described here satisfy the general requirements of the "React \& Wind" technology. 
These designs allow the bend radii to be large enough to keep the bending strain within a value that can be tolerated. Separate cable and/or short coil tests with different bend radii will be carried out in parallel to determine the degradation due to bending for various cables. At present we are investigating the following three approaches for coil ends.

\section{A. Dogbone Ends}

A mechanical model of the dogbone ends made $\sim 20$ years ago [4] is shown in Fig. 2. In order to conform to the minimum bend radius requirements, turns in the ends were first bent radially outward. Once the radius is sufficiently increased the cable is bent to the other side of the aperture. This design thus requires a reverse bend which must be handled carefully. The dogbone ends were first proposed for $\mathrm{Nb}_{3} \mathrm{Sn}$ "React \& Wind" $32 \mathrm{~mm}$ aperture SSC dipole magnets [4]. Several magnets were built with the same design with no test in between to provide feedback to the construction of the next magnet in the series. All of these magnets contained a single weakness that severely impacted the performance of all magnets. The weakness was that the cable in the reverse bend region was not constrained properly and was damaged during the construction. The magnet scientists and engineers have learned from past experience that it is not uncommon to face such problems in any magnet program whether it is based on a new design or a previously proven design. Unfortunately, no follow-up magnet was built to test the design concept after removing the above flaw in the construction.
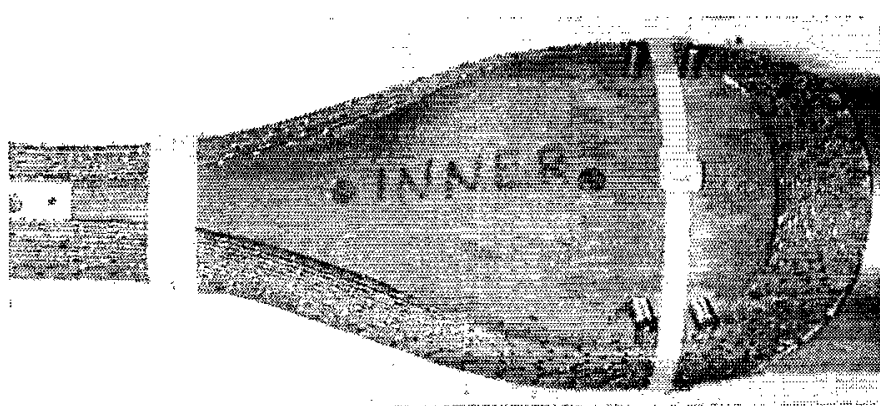

Fig. 2. Dogbone end design for "React \& Wind" technology.

Recently we have developed techniques for winding coils with a reverse bend while providing proper constraint during all phases of magnet construction. The cable is held to any general geometry with the help of a series of Kevlar strings, as shown in Fig. 3. The coil is vacuum impregnated to form a robust module. These coils would be assembled in a magnet with a structure that does not allow the strain to exceed a certain maximum value during the construction and operation of the magnet.

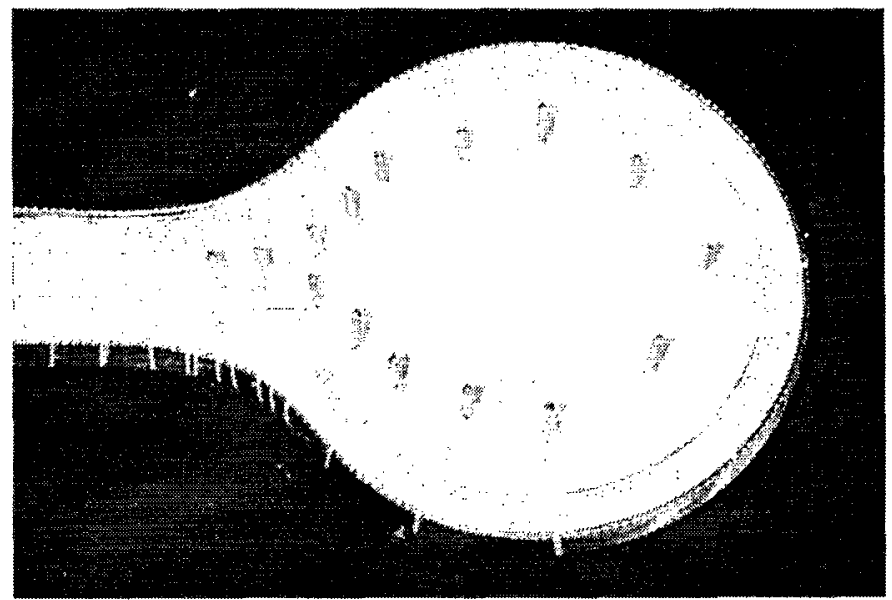

Fig. 3. Kevlar strings holding the turns tightly together in a geometry that includes ends with "reverse bend radius".

\section{B. Flared Flat Ends}

The following end designs are suitable for flat coils in a block type magnet design. The mechanical model of this general end design (which in this case looks more like a dumbbell) is shown in Fig. 3. As in dogbone ends, the cable is first bent (flared) outward, to satisfy the minimum bend radius requirements. The coil is however, kept flat throughout the winding.

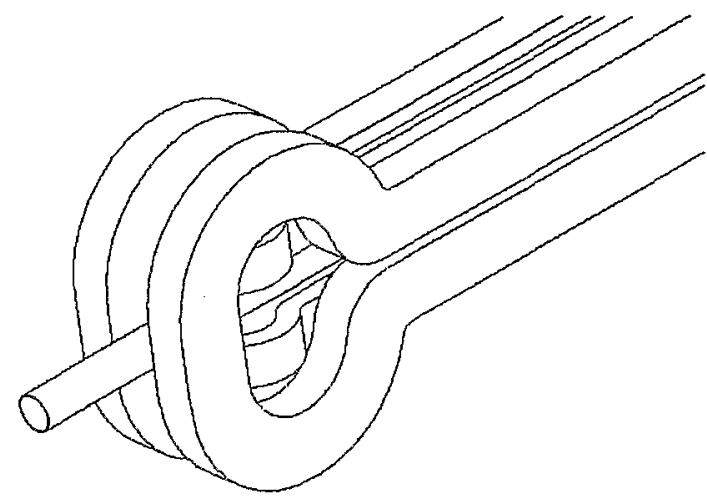

Fig. 4. Nested coil flared flat end design for "React \& Wind" technology.

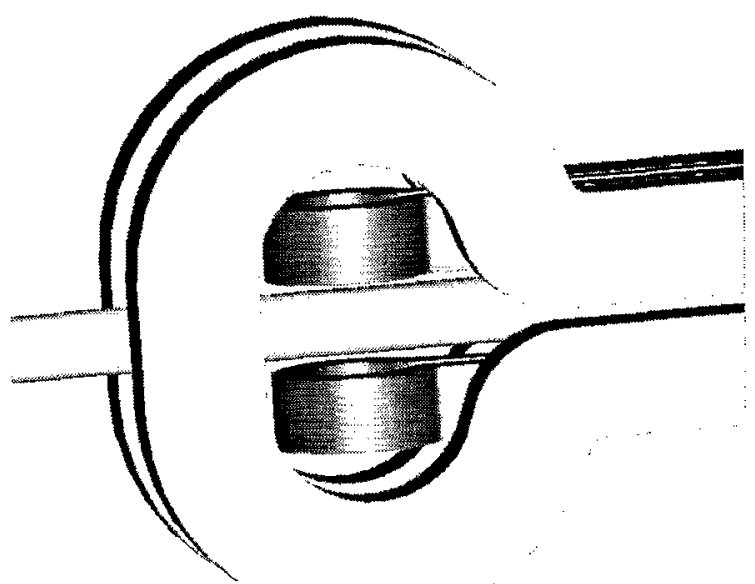

Fig. 5. Another view of the nested coil flared flat end design. 


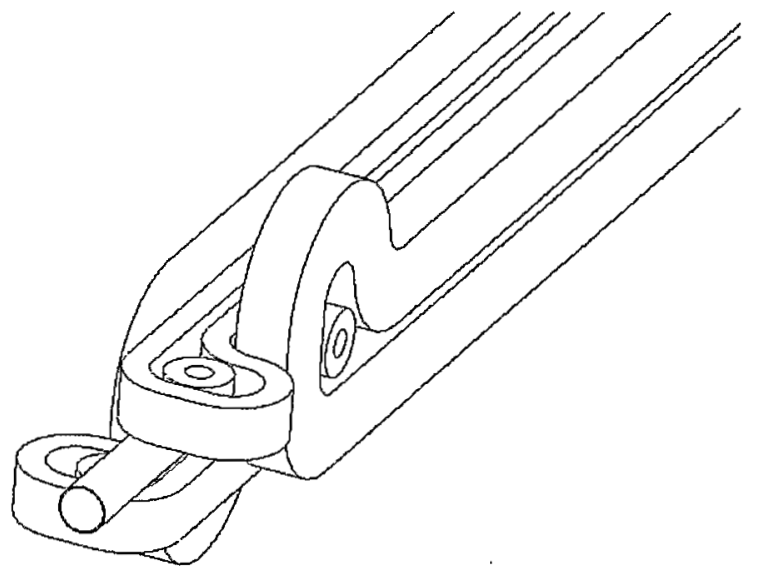

Fig. 6. Side flared flat coil end design for "React \& Wind" technology.

One needs to resolve the space conflict between the flared ends of adjacent coils when this end design is adopted for quadrupoles. This should be done in a geometry that can produce acceptable field quality. One way to accomplish this before a coil is impregnated is to lift up the flared ends until they clear each other. This would require cable to bend in the hard direction and the coil which was wound flat will no longer be flat in the ends of the magnet. To maintain the advantages of a flat coil the following two approaches to winding coil ends are proposed.

In the first design the coils are nested in the horizontal and vertical planes as shown in Fig. 4 and Fig. 5. Obviously, the coil ends need to have different radii. Despite the different lengths of coils, one should be able to obtain the required integral field quality since the coil cross sections (see Fig. 9 with description in section III) are also different and the smaller coils have longer length. In the second design, the coil ends are asymmetric to clear one side of the coil as shown in Fig. 6. A small solenoidal coil (or a racetrack coil with small straight section) is inserted inside to obtain good integrated field quality in the ends. This small coil can either be made with small diameter wire using "React \& Wind" technology or with regular "Rutherford" cable using "Wind \& React" technology.

\section{Overpass/Underpass Ends}

The proposed end design eliminates the need for reverse bends for single aperture "React \& Wind" magnets. As shown in Fig. 7, the cable is always bent in one direction or sense (either clock wise or anti-clock wise). An easy way to understand this concept is to imagine that the cable is traveling as an automobile on a highway and it has to go back to where it came from using a highway overpass/underpass bridge. One major difference between this design and the common coil design is the orientation of the cable/tape in the straight section. In this design the cable/tape is oriented parallel to the direction of field and in the case of the common coil design [3] the orientation is perpendicular to the field. This is of significant relevance to coils made with HTS tape.

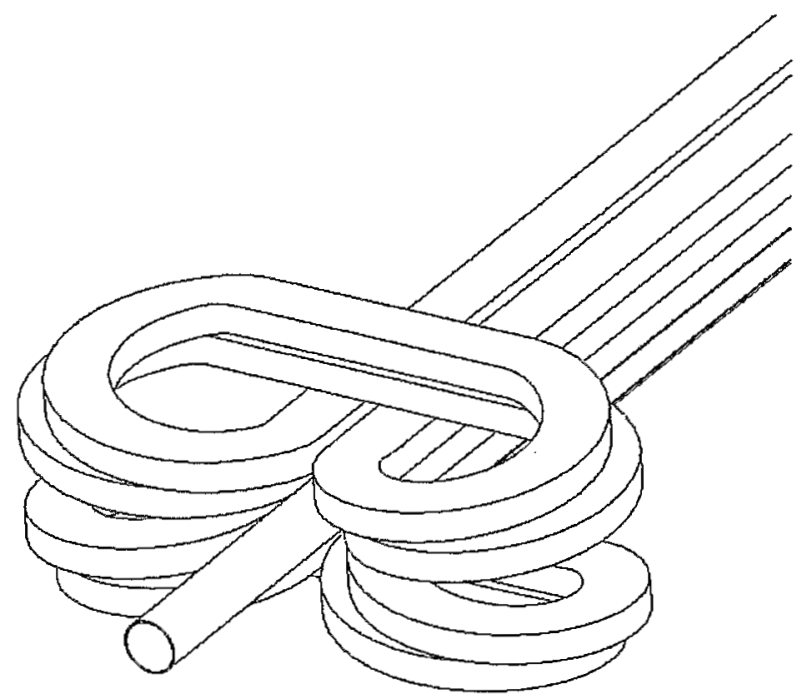

Fig. 7. Overpass/Underpass end design for "React \& Wind" technology.

\section{MAGNET CROSS SECTION DESIGNS}

At this stage of our R\&D program, we are examining both cosine theta and racetrack type coil geometries. In principle, the cosine theta type of geometry can also be produced by a set of racetrack coils. For the LHC IR upgrade, the designs must be driven by magnet construction and performance considerations instead of the conductor efficiency since only a relatively small number of magnets are needed.

\section{A. Cosine Theta Quadrupole Designs}

The quadrupole cross section shown in Fig. 8 is based on a conventional cosine theta geometry. It has an aperture of 70 $\mathrm{mm}$ and produces a gradient of $320 \mathrm{~T} / \mathrm{m}$ with state of the art $\mathrm{Nb}_{3} \mathrm{Sn}\left(\mathrm{J}_{\mathrm{c}}=2500 \mathrm{~A} / \mathrm{mm}^{2}\right.$ at $12 \mathrm{~T}$ and $\left.4.2 \mathrm{~K}\right) . \mathrm{A} 90 \mathrm{~mm}$ aperture quadrupole designed with such parameters would produce a quench gradient of $\sim 240 \mathrm{~T} / \mathrm{m}$. This cross section would use "Dogbone Ends".

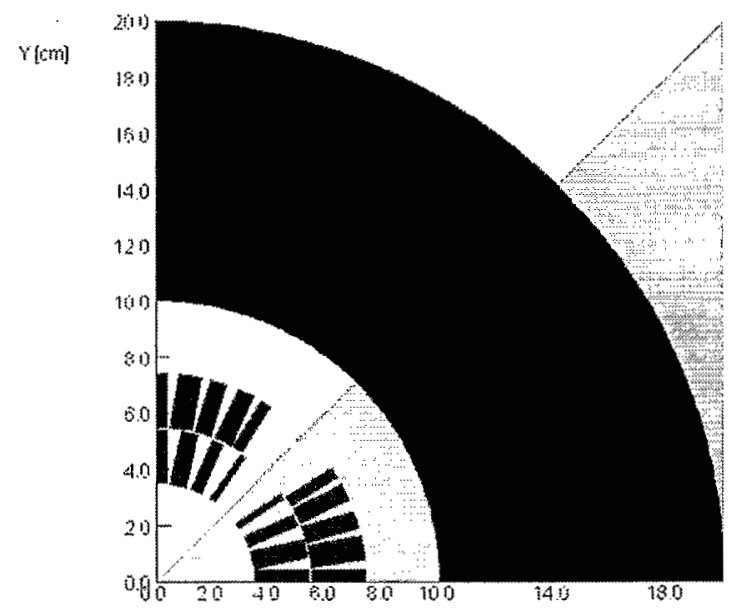

Fig. 8. A quadrant of a cosine theta quadrupole design for LHC IR upgrade.

The cross section has 8 turns in the inner layer and 14 in the outer. It is based on flat cable (zero keystone angle). One can 
get a few percent higher quench gradient in a design made with keystoned cable due to a better fill factor.

\section{B. Racetrack Coil Quadrupole Designs}

The cross-section shown in Fig. 9 is based on racetrack coil geometry. The use of two types of coils (as indicated by two shades/colors) allows the placement of conductor on the midplane while maintaining simplicity in the overall coil geometry. For this cross section, one could use either "Flared Flat Ends" or "Overpass/Underpass Ends".

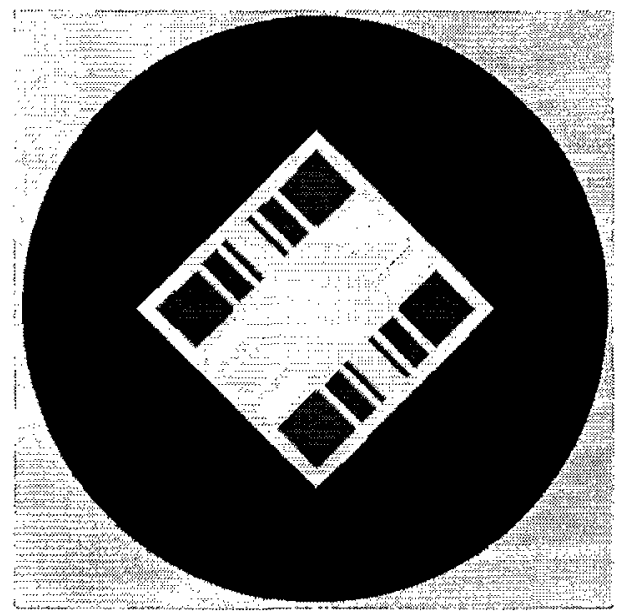

Fig. 9. A simple quadrupole concept based on two types of racetrack coils.

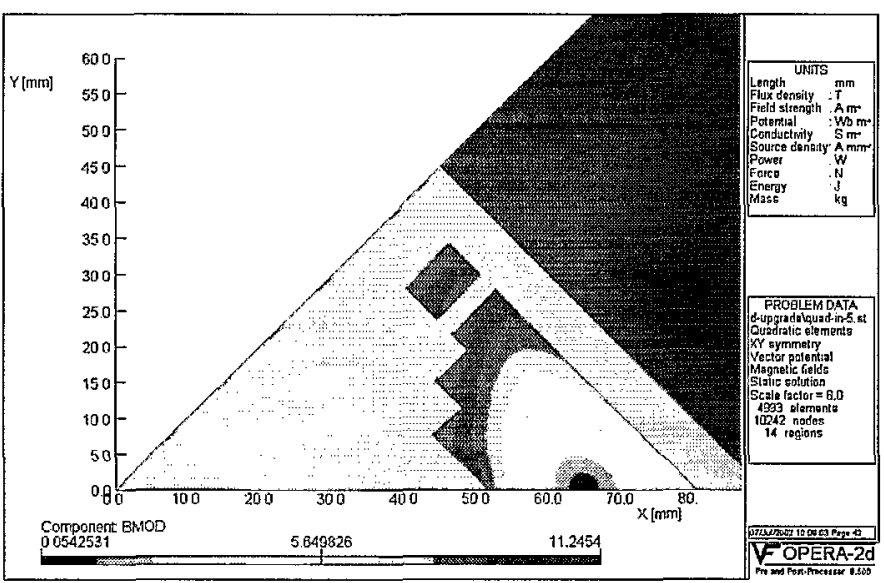

Fig. 10. An octant of the high performance quadrupole design concept based on a set of racetrack coils.

A variation of the above cross section (Fig. 9) is shown in Fig. 10 where effectively some turns from the pole region are moved to the midplane region. The design shown in Fig. 10 produces a relatively higher quench gradient as compared to the one shown in Fig. 9 because of an increased transfer function and reduced peak field. The estimated quench gradient is $\sim 230 \mathrm{~T} / \mathrm{m}$ for a $90 \mathrm{~mm}$ aperture quadrupole with $\mathrm{Nb}_{3} \mathrm{Sn} \mathrm{J}_{\mathrm{c}}$ being $2500 \mathrm{~A} / \mathrm{mm}^{2}$ at $12 \mathrm{~T}$ and $4.2 \mathrm{~K}$. This design can use either "Overpass/Underpass Ends" or "Flared Ends". In the case of the "Flared Ends", the turns in the ends of one layer may be lifted up to clear the bore tube.

\section{Racetrack Coil Dipole Designs}

React \& Wind $\mathrm{Nb}_{3} \mathrm{Sn}$ technology (also HTS technology, if a factor of 2-3 improvement in engineering current density is achieved) is well suited for LHC IR upgrade dipole. High field dipoles are being considered for "Dipole First" option [5]. A single aperture R\&D dipole with $40-80 \mathrm{~mm}$ aperture and 13 $15 \mathrm{~T}$ field is within the realm of possibility. We prefer the block type cross section. That cross section can be used with one of the three classes of end designs discussed in section. III The Overpass/Underpass ends allow a cross section where the field direction is parallel to the orientation of cable/tape. This is preferred for HTS tapes as the current density in this orientation is significantly larger.

\section{MAGNET R\&D APPROACH}

The magnet $\mathrm{R} \& \mathrm{D}$ approach of developing quadrupoles for an LHC IR upgrade would be similar to and in continuation of our present common coil magnet program [2]. In fact most of the experience and tooling developed for common coil program will be utilized here. We will systematically and experimentally evaluate various designs and technology using a rapid turn around program.

\section{SUMMARY AND CONCLUSION}

The "React \& Wind" approach is attractive for building high field accelerator magnets as it eliminates the need of subjecting the whole coil module to high reaction temperature. The R\&D program presented here will systematically address the issues related to this technology with the goal of building prototype LHC IR upgrade magnets for LHC IR upgrade. Several design concepts have been presented here that allow the use of brittle materials in building high field magnets using a "React \& Wind" approach. The magnet development program at BNL is geared for building scalable long magnets.

\section{ACKNOWLEDGMENT}

The authors wish to acknowledge the benefits of discussions from F. Pilat, S. Peggs and E. Willen at BNL.

\section{REFERENCES}

[1] L. Rossi, "The LHC Main Dipoles and Quadrupoles toward Series Production," these proceedings.

[2] M. Anerella, et al., "Magnet Engineering and Test Results of High Field Magnet Program at BNL," these proceedings.

[3] R. Gupta, "A Common Coil Design for High Field 2-in-1 Accelerator Magnets," Proc. 1997 Particle Accelerator Conf., p. 3344.

[4] J. G. Cottingham., "Test Results from Two 5m Two-in-One Superconducting Magnets for the SSC", IEEE Trans. on Magnetics, Vol. MAG-21, No. 2, March 1985, p. 1018.

[5] O. Bruining, CERN, LHC Upgrade Collaboration Meeting, March 11 12, 2002. Organisor: F. Ruggiero, http://cern.ch/lhc-proj-IR-upgrade.

[6] R. Gupta et al., "Field Quality Optimization in a Common Coil Magnet Design," IEEE Trans. Applied Superconductivity, Vol. 10, No. 1, March 2000, p. 326. 